\title{
Comunidad Colombiana de Educación Matemática: una caracterización documental
}

\section{Colombian Community of Mathematics Education: a documentary characterization}

\author{
Paola Castro* \\ ORCID iD 0000-0002-3333-2461 \\ Pedro Gómez ${ }^{* *}$ \\ ORCID iD 0000-0001-9929-4675 \\ Sileni-Marcela Carranza ${ }^{* * *}$ \\ ORCID iD 0000-0002-5477-9078 \\ María C. Cañadas ${ }^{* * * *}$ \\ ORCID iD 0000-0001-5703-2335
}

\begin{abstract}
Resumen
Caracterizamos la comunidad colombiana de Educación Matemática desde su documentación de acceso abierto. Analizamos del contenido de 3475 documentos publicados entre 1983 y 2017, con una aproximación semántica, desde una taxonomía específica para la Educación Matemática. Describimos el comportamiento diacrónico de la documentación y establecemos la especialidad temática, la evolución en el tiempo de los contenidos a través de los conjuntos de variables nivel educativo, nociones pedagógicas y temas de matemáticas, y la asociación entre estos conjuntos. Los resultados ratifican que la Educación Matemática en Colombia es un frente de estudio. Los focos de interés de la comunidad son los niveles educativos pregrado y media, las nociones pedagógicas aprendizaje, aula y enseñanza, y los temas de matemáticas geometría, álgebra y números. Este estudio deja abierta la discusión respecto al papel de la investigación y la innovación en la consolidación de la Educación Matemática como disciplina.
\end{abstract}

Palabras clave: Análisis documental. Bibliometría. Comunidad. Indexación de materias. Educación matemática.

\footnotetext{
* Candidata a Doctora en Educación, Universidad de los Andes. Gestora académica, Universidad de los Andes, Bogotá, Colombia. Dirección postal: Calle 18A \#0-19 Este, 111711, Bogotá, Colombia. E-mail: dp.castro116@uniandes.edu.co.

${ }^{* *}$ Doctor en Matemáticas, Universidad de Granada (UGR). Profesor visitante, Universidad de los Andes, Bogotá, Colombia. Dirección postal: Calle 18A \#0-19 Este, 111711, Bogotá, Colombia. E-mail: argeifontes@ gmail.com. **** Licenciada en Matemáticas, Universidad Pedagógica Nacional (UPN). Docente, Gimnasio Bilingüe Obregón, Bogotá, Colombia. Dirección postal: Calle 62 A Sur No. 67-08, 111911, Bogotá, Colombia. E-mail: sileni.carranza@ hotmail.com.

***** Doctora en Didáctica de la Matemática, Universidad de Granada (UGR). Profesora titular, Universidad de Granada, Granada, España. Dirección postal: Campus Universitario de la Cartuja, s/n, 18071, Granada, España. E-mail: mconsu@ugr.es.
} 


\begin{abstract}
We characterize the Colombian research and innovation community in Mathematics Education from its open access documentary production. We analyzed the content of 3475 documents published by this community between 1983 and 2017, with a semantic approach, from a taxonomy of key terms specific to Mathematics Education. We analyzed the diachronic behavior of the documentation and established the thematic specialty, the evolution of the content over time through the sets of variables such as educational level, pedagogical notions, and mathematics topics, and the association between these sets. The results confirm that Mathematics Education in Colombia is a study front. The focal points of interest for the community are the undergraduate and secondary educational levels, the pedagogical notions of learning, classroom and teaching, and the topics of geometry, algebra, and numbers. This study leaves an open discussion regarding the role of research and innovation in the consolidation of Mathematics Education as a discipline.
\end{abstract}

Keywords: Document analysis. Bibliometrics. Community. Subject index terms. Mathematics Education.

\title{
1 Introducción
}

Desde una aproximación social a la definición de disciplina, podemos afirmar que la Educación Matemática se ha consolidado como disciplina científica en Colombia. Una disciplina surge cuando existe una comunidad con un foco de interés, un lenguaje y un propósito común (ERNEST, 1998). En Colombia, la Educación Matemática ha llegado a ser investigada y enseñada de manera importante. Como resultado de esto, se identifican publicaciones académicas específicas y se desarrollan encuentros de carácter internacional, nacional y regional en torno a ella. Se percibe un crecimiento importante de la producción documental en esta disciplina como resultado de la consolidación de una comunidad de investigadores y educadores matemáticos, por lo que resulta relevante hacer un balance de esta producción respecto a sus focos de interés.

Hasta el momento, en Colombia se han realizado algunos estudios relacionados con eventos académicos (por ejemplo, CASTRO; GÓMEZ, 2017; BONILLA; OBANDO, 2018), pero no se identifican investigaciones que den cuenta del comportamiento de la globalidad de la producción en Educación Matemática en el país, ni de los énfasis de trabajo de la comunidad. Su documentación es diversa y se difunde en diferentes medios. Por ello, resulta relevante analizar la variedad de trabajos de acceso abierto, tanto de investigación como de innovación curricular, porque, en una disciplina educativa, el conocimiento que surge de la investigación y de la innovación debe articularse. Debe promoverse, por un lado, el uso de la investigación y, por otro, el reconocimiento del saber práctico (MUÑOZ-REPISO, 2010). Es importante hacer un análisis de qué se ha hecho y qué no en ambos ámbitos, para poder profundizar en el conocimiento existente o para iniciar nuevas líneas de estudio. 
Para reafirmar el estatus científico de la Educación Matemática en una comunidad y detectar sus focos de interés, se debe partir de la diversidad de su documentación (BRACHO et al., 2014). Identificar los focos de interés de la comunidad proporciona información sobre su estado actual en relación con los fenómenos y problemas que aborda.

En Educación Matemática, se identifica una cantidad importante de estudios documentales que sus autores presentan como bibliométricos o cienciométricos. La mayoría de estos trabajos se han desarrollado en España. Su énfasis ha estado en los indicadores de productividad e impacto (por ejemplo, VALLEJO-RUIZ et al., 2006; MAZ et al., 2009). Pocos estudios abordan el análisis de la documentación a partir de un marco teórico específico de la Educación Matemática (GÓMEZ et al., 2011; BRACHO et al., 2014; MAZ-MACHADO et al., 2015).

Caracterizar la comunidad colombiana de Educación Matemática implica analizar su comportamiento diacrónico e identificar sus focos de interés. Describir la producción documental de una disciplina como esta supone estudiar la diversidad de sus documentos e identificar los fenómenos que aborda la comunidad. Es conveniente partir de categorías deductivas - establecidas desde enfoques teóricos propios de la disciplina y fundamentadas en ellos - para identificar los temas tratados en los documentos. Estas categorías, aunque se plantean antes del análisis de la información, permiten identificar los temas tratados a partir de un esquema de normalización en la asignación de los descriptores que los distinguen. Las categorías son la base para organizar la documentación que se estudia.

Caracterizamos la comunidad colombiana de Educación Matemática a partir del análisis del contenido de la documentación de acceso abierto que fue producida entre 1983 y 2017. Los documentos que conforman la muestra fueron difundidos de manera abierta o compartidos con el equipo investigador por asociaciones, revistas y autores colombianos de manera autónoma.

\section{Marco conceptual}

Estructuramos el marco conceptual en tres partes: (a) la relación entre comunidad y disciplina, (b) las generalidades de la cienciometría que son relevantes en este estudio y (c) la presentación de una propuesta de taxonomía de términos específicos de la Educación Matemática de la que surgen las variables de interés. 


\subsection{Comunidad y disciplina}

Desde el enfoque sociológico, la Educación Matemática es una disciplina que está consolidada en y por una comunidad, académica y de práctica, con espacios propios de comunicación y de difusión (ROMBERG, 1992; ERNEST, 1998). Además de contar con programas de formación específicos, en ella, se identifican agremiaciones que lideran reuniones periódicas (congresos, coloquios, jornadas, encuentros etc.) y publicaciones especializadas. En la comunidad colombiana de Educación Matemática, se emplean diversos medios de divulgación de sus resultados (WALDEGG, 1998). Así, se publican artículos y libros que resultan de investigaciones, se produce documentación que surge de encuentros periódicos de investigadores y educadores (memorias y presentaciones), documentación no publicada de grupos de investigación o autores, trabajos de grado de licenciaturas y tesis de posgrados, y actividades de enseñanza, entre otros. Por lo tanto, proporcionar información de la documentación en relación con las tendencias temáticas y su comportamiento en el tiempo permite caracterizar la comunidad a partir de los fenómenos y problemas que aborda.

\subsection{Cienciometría}

La cienciometría se define como la ciencia que permite cuantificar actividades científicas en una disciplina (MACÍAS-CHAPULA, 2001). Esta ciencia se interesa por el crecimiento cuantitativo de la actividad científica, la productividad de los investigadores $\mathrm{y}$, en general, por su desarrollo (SPINAK, 1996). Incluye el análisis de factores que pueden ser concluyentes en el desarrollo de la actividad científica de la disciplina (PÉREZ-ANGÓN, 2006), pues proporciona información sobre la cantidad de investigadores, las fuentes de financiación, la distribución por especialidad (asociada al contenido), la distribución geográfica y su productividad diacrónica. La cienciometría emplea procedimientos matemáticos y análisis estadísticos para investigar las características de la investigación científica (ARENCIBIA; DE MOYA, 2008). Por tanto, puede verse como una posibilidad para evaluar la producción científica que ofrece una visión panorámica y cuantificada de esta producción en un contexto, un tiempo y un campo científico (MEDINA, 2005).

Las leyes cienciométricas actúan como criterios normativos que describen el comportamiento de los procesos de producción científica (MILLÁN et al., 2018). Estas leyes nos permiten identificar comportamientos estadísticamente regulares en el tiempo en relación con la producción y el consumo de la información científica (ARDANUY, 2012). 
La ley de crecimiento exponencial se concibe como una regla fundamental para cualquier análisis de la ciencia (PRICE, 1973). El crecimiento de la ciencia tiene un comportamiento exponencial, de modo que la tasa de crecimiento es proporcional al tamaño de la población o magnitud total adquirida. De acuerdo con la ley de Price, la evolución de cada disciplina se da en las siguientes etapas: precursores, crecimiento exponencial y crecimiento lineal (ARDANUY, 2012). En la etapa de precursores, se dan las primeras publicaciones de la disciplina. En la etapa de crecimiento exponencial, la disciplina se convierte en un frente de estudio. En la etapa de crecimiento lineal, el crecimiento de la producción se desacelera.

La bibliometría estudia la ciencia a partir de las fuentes bibliográficas, con el propósito de identificar sus autores, sus relaciones y sus tendencias (SPINAK, 2001). Además, la bibliometría considera los elementos representativos de la documentación, tales como autores, título de la publicación, tipo de documento, idioma, resumen y palabras claves (SOLANO et al., 2009). Aunque los artículos de investigación son materia prima principal en la aproximación bibliométrica al estudio de la ciencia, esto no implica que se dejen de lado otros documentos que merecen ser analizados (CALLON et al., 1995), como comunicaciones a congresos u otras reuniones científicas, libros, monografías, tesis, memorias, curso o seminarios.

Los indicadores bibliométricos son los datos numéricos con los que se busca representar fenómenos sociales de la actividad científica a partir de la comunicación escrita de una disciplina (LÓPEZ-PIÑERO; TERRADA-FERRANDIS, 1992). Estos indicadores permiten evaluar, determinar e informar sobre los resultados del crecimiento en el proceso investigativo en cualquier campo de la ciencia (ESCORCIA, 2008). Los indicadores bibliométricos pueden ser de publicación y citación, de actividad (producción, visibilidad e impacto, y colaboración), de especialización temática y de calidad, impacto y asociaciones temáticas. En este estudio, usamos los siguientes indicadores de producción: productividad diacrónica, evolución diacrónica de los contenidos, índice de especialización temática y de asociaciones temáticas.

La productividad diacrónica evidencia el dinamismo de una disciplina en el tiempo. $\mathrm{Su}$ análisis permite verificar si la evolución de la disciplina responde a la ley del crecimiento de la investigación y en qué etapa se ubica (PRICE, 1973). Los indicadores que permiten ahondar en los contenidos y especialización temática de una disciplina se asocian al análisis del contenido, en especial si se hace desde una clasificación de términos específicos. El análisis de contenido es una técnica que permite formular, a partir de ciertos datos, inferencias válidas que puedan aplicarse a su contexto (KRIPPENDORFF, 1990). La lectura y la organización de los datos se puede desarrollar a partir de unas categorías que estén relacionadas con bloques temáticos o materias que se encuentran en tesauros estandarizados, o que surgen de clasificaciones del área 
objeto de estudio (FERNÁNDEZ-CANO; BUENO-SÁNCHEZ, 1998). Además de establecer la especialización temática, es posible identificar si hay asociaciones entre los temas tratados en la documentación (SANCHO, 1990).

\subsection{Términos específicos en Educación Matemática}

Para este estudio, seleccionamos la taxonomía de términos clave, específica a la Educación Matemática, propuesta por Gómez y Cañadas (2013). Esta taxonomía está basada en un estándar para la construcción, formato y gestión de vocabularios controlados (NISO, 2005). Tras evaluar los tesauros de la UNESCO y ERIC, que resultaron limitados para la Educación Matemática, los autores construyeron su propia taxonomía de términos clave basados en MathEduc (FIZ KARLSRUHE, 2010), de modo que todo término clave de esa base de datos tuviera un término equivalente en su propuesta. Gómez y Cañadas (2013) definen un conjunto de categorías que reúnen los términos clave de la Educación Matemática.

La categoría asociada al nivel educativo se centra en el tipo de formación que trata el documento: educación infantil, educación primaria, educación secundaria, educación media, título de grado universitario, estudios de posgrado, formación profesional, educación de adultos, todos los niveles educativos, otro nivel educativo y ningún nivel educativo.

La taxonomía se basa en un marco conceptual específico de la Educación Matemática y en un enfoque curricular que aborda cuatro cuestiones centrales: el conocimiento a enseñar, el aprendizaje, los métodos de enseñanza y la valoración de los aprendizajes realizados. A partir de esta teoría, los autores proponen la categoría de teoría curricular que contiene términos clave asociados con sistema educativo, centro educativo, aula, alumno, profesor, aprendizaje, enseñanza, evaluación y currículo. Los autores incluyen otros términos clave en esta categoría: otras nociones en Educación Matemática, Educación Matemática y otras disciplinas, e investigación e innovación en Educación Matemática. Por la diversidad en la naturaleza de los términos de la categoría de teoría curricular, nos referimos a ellos como nociones pedagógicas.

Los autores diferencian las matemáticas escolares de las matemáticas superiores. La categoría de matemáticas escolares incluye los contenidos de cálculo, estadística, geometría, medida, números, probabilidad, álgebra y otros temas de matemáticas escolares. La categoría de matemáticas superiores incluye contenidos de álgebra, análisis, combinatoria, cálculo, ecuaciones diferenciales, estadística, geometría, lógica matemática, matemática discreta, probabilidad, teoría de conjuntos, teoría de grafos, teoría de la medida, teoría de números, topología y otros temas de matemáticas superiores. 
Con base en las categorías de la taxonomía descrita previamente, realizamos el análisis del contenido de la documentación producida por la comunidad colombiana de Educación Matemática. Establecemos sus características en términos de los niveles educativos, las nociones pedagógicas y los temas de matemáticas que aborda en su producción documental.

\section{Objetivos}

El objetivo del estudio es caracterizar la comunidad colombiana de Educación Matemática desde su documentación de acceso abierto producida entre 1983 y 2017. Para su consecución, definimos los siguientes objetivos específicos.

- Establecer el comportamiento diacrónico de la cantidad de documentos.

- Determinar la medida en la que se tratan los niveles educativos, las nociones pedagógicas y los temas de matemáticas.

- Describir la evolución diacrónica de los contenidos de la documentación, en términos de nivel educativo, nociones pedagógicas y temas de matemáticas.

- Establecer si existe asociación entre las categorías nivel educativo, nociones pedagógicas y temas de matemáticas.

\section{Método}

Este estudio es un estudio de caso, documental de tipo descriptivo y cienciométrico. Seguidamente, presentamos las fuentes de información y los procedimientos.

\subsection{Fuentes de información}

La población del estudio es la producción documental digital de acceso abierto de la comunidad de Educación Matemática, que es divulgada a través de diversos canales de difusión colombianos como revistas, eventos y repositorios institucionales. En este estudio, analizamos todos los documentos que pudimos obtener de las fuentes que componen la población. Por consiguiente, contamos con la documentación de la población para la que no se restringe el acceso. La muestra corresponde a 3475 documentos, publicados de manera digital entre 1983 y 2017. No encontramos documentación en formato digital que fuera publicada antes de 1983. 
El proceso de búsqueda de la documentación fue sistemático, inició en 2014 y terminó en 2018. Buscamos e identificamos universidades y asociaciones de investigadores y educadores que desarrollan trabajos en Educación Matemática en Colombia. Accedimos a la documentación de Educación Matemática que es difundida de manera abierta en páginas web de eventos académicos y revistas, y en repositorios institucionales de universidades, grupos de investigación e instituciones gubernamentales y no gubernamentales. Adicionalmente, contactamos con las personas responsables de gestionar eventos y publicaciones en el país, cuya documentación no está disponible en la web, con el propósito de obtener su autorización expresa para acceder a sus documentos.

Los documentos de Educación Matemática que fueron analizados corresponden a memorias de veinte eventos de docentes e investigadores liderados por agremiaciones que difundieron su documentación de manera abierta, o que manifestaron su autorización para que accediéramos a ella. Algunos de estos eventos son el Encuentro Colombiano de Matemática Educativa, el Encuentro de Geometría y sus Aplicaciones de Colombia, el Encuentro Nacional de Educación Matemática y Estadística, y el Coloquio Regional de Matemáticas y Simposio de Estadística. Así mismo, tomamos artículos de 24 revistas editadas en Colombia que han publicado trabajos de investigación e innovación curricular en Educación Matemática. De estas revistas, cuatro son específicas en la disciplina (Revista EMA, Revista Ejes, Revista Latinoamericana de Etnomatemática y Revista Colombiana de Matemática Educativa).

Accedimos a trabajos de grado de licenciatura y tesis de posgrados (especialización, maestría y doctorado) en Educación Matemática difundidos en repositorios institucionales de doce universidades públicas y privadas. Otros documentos corresponden a libros, capítulos de libros y avances de trabajos o resultados de investigación compartidos por investigadores y educadores matemáticos de manera autónoma.

En la muestra, contamos con documentación producida por autores no colombianos, que fue divulgada en canales de difusión propios de la comunidad colombiana de Educación Matemática. No descartamos las publicaciones de autores colombianos en contextos internacionales; sin embargo, no las incluimos en el proceso de búsqueda. No obstante, algunos de estos autores compartieron sin restricciones con el equipo investigador las contribuciones que no fueron publicadas en revistas del país.

Aunque el muestreo no es probabilístico, respaldamos su representatividad debido al proceso de búsqueda de las fuentes de información y a la diversidad de los documentos que se encuentran en ella. Incluimos actas de eventos, comunicaciones, conferencias, talleres, artículos de revistas, libros y capítulos de libros, trabajos de grado y tesis, y recursos de enseñanza. En 
la Tabla 1, mostramos la cantidad de documentos analizados de acuerdo con su tipo y la proporción a la que corresponde esta cantidad según el tamaño de la muestra.

Tabla 1 - Distribución de documentos por tipo

\begin{tabular}{lcc}
\hline \multicolumn{1}{c}{ Tipo de documento } & Cantidad & Proporción \\
\hline Acta de evento & 1396 & $40,2 \%$ \\
Artículo & 791 & $22,8 \%$ \\
Capítulo de libro & 455 & $13,1 \%$ \\
Tesis de posgrado & 270 & $7,8 \%$ \\
Conferencia o comunicación & 254 & $7,3 \%$ \\
Trabajo de grado de licenciatura & 211 & $6,1 \%$ \\
Documento no publicado & 52 & $1,5 \%$ \\
Libro & 41 & $1,2 \%$ \\
\hline
\end{tabular}

Fuente: elaborada por los autores

\subsection{Variables}

Definimos los conjuntos de variables del estudio de acuerdo con las categorías de la taxonomía que describimos en el marco conceptual. Los conjuntos de variables son nivel educativo, nociones pedagógicas y temas de matemáticas.

En primer lugar, adaptamos las etiquetas de la taxonomía (GÓMEZ; CAÑADAS, 2013) que se refieren al nivel educativo de acuerdo con el contexto colombiano. Adoptamos las etiquetas del contexto colombiano como las variables del conjunto nivel educativo de este estudio (Cuadro 1).

\begin{tabular}{|l|l|}
\hline Taxonomía (GÓMEZ; CAÑNADS, 2013) & Contexto colombiano (variables) \\
\hline Educación infantil (0 a 6 años) & Preescolar \\
Educación primaria (7 a 12 años) & Primaria \\
Educación secundaria (13 a 16 años) & Secundaria \\
Educación media (17 y 18 años) & Educación media \\
Educación de adultos & Educación de adultos \\
Formación profesional & Formación continua/técnica \\
Título de grado universitario & Pregrado \\
Estudios de posgrado & Posgrado \\
Todos los niveles educativos & Todos los niveles (en general) \\
Otro nivel educativo & Otro nivel educativo \\
Ningún nivel educativo & Ningún nivel educativo \\
\hline
\end{tabular}

Cuadro 1 - Variables de nivel educativo

Fuente: elaborado por los autores

En cuanto a las nociones pedagógicas, agregamos los términos análisis de contenido y resolución de problemas debido a la importancia que se percibe de ellos en la documentación. Por tanto, el conjunto nociones pedagógicas está configurado por las siguientes variables: sistema educativo, centro educativo, aula, alumno, profesor, aprendizaje, enseñanza, evaluación, gestión curricular, análisis de contenido (historia de los contenidos, sistemas de representación y fenomenología), resolución de problemas, otras disciplinas, metodología de investigación y otras nociones. Pese a que la variable metodología de investigación no debería 
ser concebida como una noción pedagógica, la incluimos en este conjunto de variables con el propósito de verificar, con los resultados del estudio, su relevancia en comparación con otras nociones.

Para aludir a los temas de matemáticas, proponemos siete variables que reúnen los términos de las matemáticas escolares y las matemáticas superiores incluidos en la taxonomía. Incluimos una octava variable denominada cualquier tema. Asociamos esta variable a los documentos que no especifican un contenido matemático, pero que abordan cuestiones que son aplicables a cualquier tema (Cuadro 2).

\begin{tabular}{|c|c|c|}
\hline Variables & Matemáticas escolares & Matemáticas superiores \\
\hline Álgebra & Álgebra & $\begin{array}{l}\text { Álgebra, lógica matemática y } \\
\text { teoría de grafos }\end{array}$ \\
\hline Cálculo & Cálculo & $\begin{array}{l}\text { Análisis, cálculo y ecuaciones } \\
\text { diferenciales }\end{array}$ \\
\hline Estadística y probabilidad & Estadística y probabilidad & $\begin{array}{l}\text { Combinatoria, estadística y } \\
\text { probabilidad }\end{array}$ \\
\hline Geometría & Geometría & Geometría y topología \\
\hline Medida & Medida & Teoría de la medida \\
\hline Números & Números & Teoría de números \\
\hline Otros temas & Otros temas & $\begin{array}{l}\text { Matemática discreta, teoría de } \\
\text { conjuntos y otros temas }\end{array}$ \\
\hline Cualquier tema & & \\
\hline
\end{tabular}

Fuente: elaborado por los autores

\subsection{Procedimientos}

Realizamos el análisis del contenido de la documentación con una aproximación semántica con el propósito de codificar los documentos en términos de las variables anteriores. Asignamos las variables que le corresponden a los documentos luego de su lectura, con el detalle necesario para identificar los fenómenos y problemas concretos que abordan. Organizamos los resultados de la codificación en bases de datos. Un documento podía ser codificado en una o más variables de los conjuntos nivel educativo, nociones pedagógicas y temas de matemáticas. Para los tres conjuntos de variables, hay un éxito si el documento está codificado en una variable (valor 1) y un fracaso, si no lo está (valor 0).

Un equipo de codificadores, con título de grado en Educación Matemática, registró la información bibliográfica de cada documento (título, resumen, autores, año) y determinó su enfoque. Luego, identificó términos clave que describen el contenido del documento, que se corresponden con variables de los conjuntos nivel educativo, nociones curriculares y temas de matemáticas. Una vez realizada la codificación, un revisor de las codificaciones, máster en Educación Matemática, verificó la validez y precisión de la información que se registró para 
cada documento. Por último, un segundo revisor, doctor en Educación Matemática, revisó aleatoriamente el trabajo realizado por el revisor de la codificación.

Presentamos, a continuación, los procedimientos que realizamos para analizar los datos de acuerdo con los objetivos de investigación.

\subsubsection{Comportamiento diacrónico de la documentación}

Con el propósito de establecer el comportamiento diacrónico de la producción documental, utilizamos gráficos de líneas para representar la cantidad de documentos producidos por año. Determinamos el modelo que mejor se ajusta a los datos, de acuerdo con el coeficiente de determinación. De esta forma, verificamos si los documentos de la muestra satisfacen la ley de crecimiento (PRICE, 1973).

\subsubsection{Especialización temática}

Determinamos la medida en la que se tratan las variables de cada conjunto. Definimos el índice de especialización de cada variable como el cociente entre la cantidad de documentos que trata la variable y el total de documentos de la muestra. Identificamos los focos de interés de la comunidad respecto al indicador bibliométrico de especialización temática en términos de cada variable de los conjuntos nivel educativo, nociones pedagógicas y temas de matemáticas.

\subsubsection{Comportamiento diacrónico de los contenidos}

Empleamos gráficos de barras apiladas para identificar el comportamiento diacrónico de los contenidos en cada conjunto de variables. De acuerdo con las proporciones en las que se tratan las diferentes variables, identificamos si los focos de interés de la comunidad han cambiado en el tiempo o si se conservan. Para confirmar si el comportamiento de cada variable en el tiempo evidencia o no alguna tendencia, graficamos la serie temporal correspondiente. Realizamos la prueba de Phillips-Perron (PP) para rechazar o no la hipótesis nula de que cada serie no es estacionaria en media. 


\subsubsection{Independencia entre conjuntos de variables}

Por último, utilizamos tablas de contingencia y realizamos pruebas de independencia para establecer si hay asociación entre las variables de los siguientes pares de conjuntos de variables: (a) nivel educativo y nociones pedagógicas, (b) nivel educativo y temas de matemáticas, y (c) nociones pedagógicas y temas de matemáticas.

\section{Resultados}

Para dar cumplimiento a los objetivos del estudio, organizamos los resultados en cinco secciones. Primero, analizamos el comportamiento diacrónico de la documentación. Luego, exponemos los índices de especialización temática de la comunidad respecto a las variables de los conjuntos nivel educativo, nociones pedagógicas y temas de matemáticas. Describimos el comportamiento diacrónico de los contenidos de la documentación en términos de las mismas variables y comprobamos si existe independencia entre los cuatro conjuntos de variables. Por último, presentamos un resumen de los resultados.

\subsection{Comportamiento diacrónico de la documentación}

De acuerdo con la muestra, representamos en la Figura 1 la cantidad de documentos que fueron producidos entre 1983 y 2017. Buscamos el modelo que mejor relaciona la cantidad de documentos con la variable tiempo, de acuerdo con el coeficiente de determinación. Encontramos que la línea de tendencia que mejor se ajusta es la de tipo exponencial. El coeficiente de determinación de este modelo es $\mathrm{R}^{2}=0,9145$, lo que indica que el $91 \%$ de la variación en la cantidad de publicaciones se explica por la variación en el tiempo. La cantidad de documentos tiene una tasa de crecimiento de veinte documentos por año.

De acuerdo con la ley de crecimiento (PRICE, 1973), podemos establecer que la Educación Matemática en Colombia se consolida como un frente de estudio debido al crecimiento exponencial de documentación. Entre 1983 y 1994, identificamos un comportamiento que se puede asociar con la etapa de precursores (ARDANUY, 2012). Observamos algunos descensos en la línea de producción documental en los años 2009, 2010 y 2017. En 2009 y 2010 hubo una reducción importante de contribuciones a eventos. En 2013, una revista no especializada en la disciplina produjo una edición especial exclusiva para Educación Matemática. Esto explica la reducción que se dio en 2014 en la cantidad de artículos 
publicados. El descenso que se observa en 2017 se explica porque no se realizaron algunos eventos relevantes en la comunidad, como el Encuentro Colombiano de Matemática Educativa.

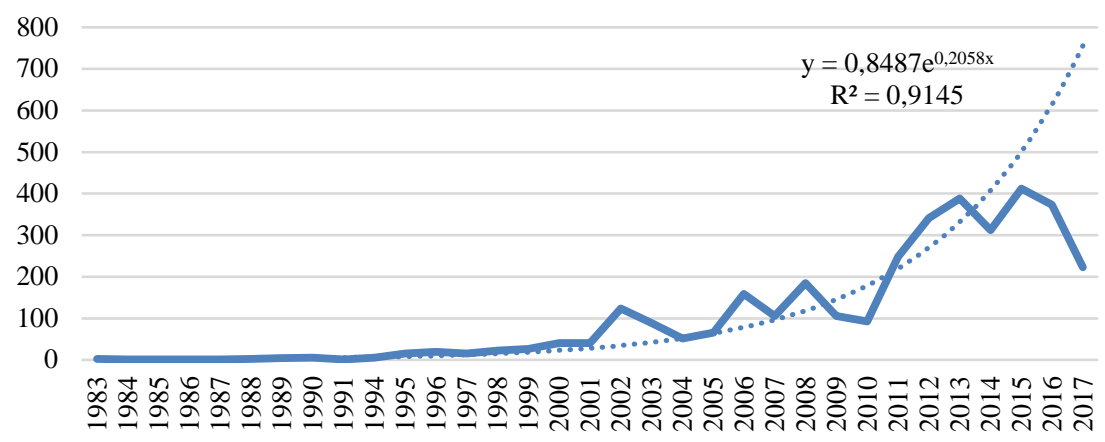

Figura 1 - Comportamiento diacrónico de la documentación Fuente: elaborada por los autores

\section{2 Índice de especialización temática}

Presentamos el índice de especialización temática de acuerdo con las variables de los conjuntos de variables del estudio. Definimos el índice de especialización de cada variable como el cociente entre la cantidad de documentos que fue codificado en cada una de las variables y el total de documentos de la muestra.

En la Figura 2, presentamos la organización jerárquica de los niveles educativos de acuerdo con la medida en la que se tratan en la muestra.

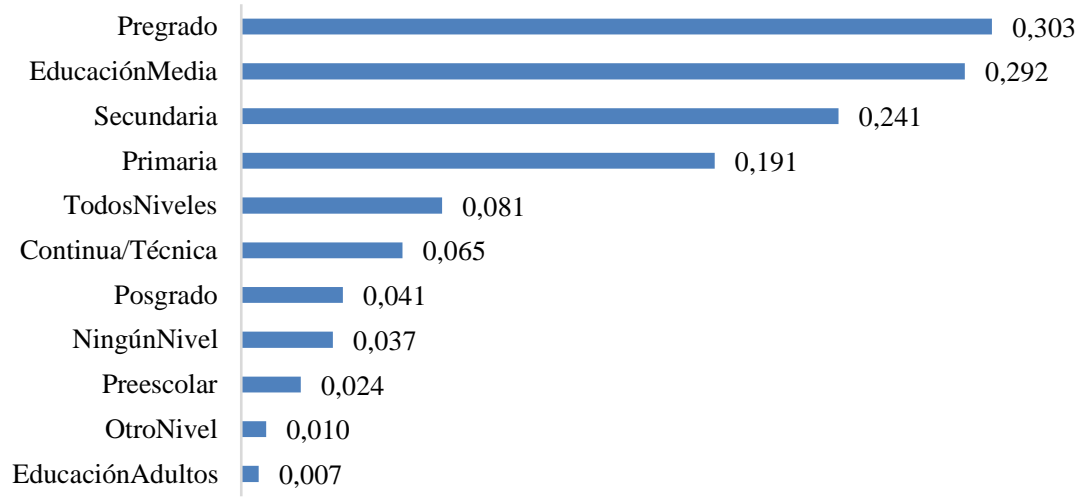

Figura 2 - Índice de especialización temática en nivel educativo Fuente: elaborada por los autores

Los niveles educativos que más trata la comunidad son pregrado, educación media, secundaria y primaria. Sus índices superan, de manera evidente, los índices que tienen los niveles educativos de preescolar, educación de adultos, formación continua/técnica y posgrado. Resulta importante resaltar el índice tan reducido que tiene la educación inicial (preescolar) en relación con los otros niveles de la educación básica. 


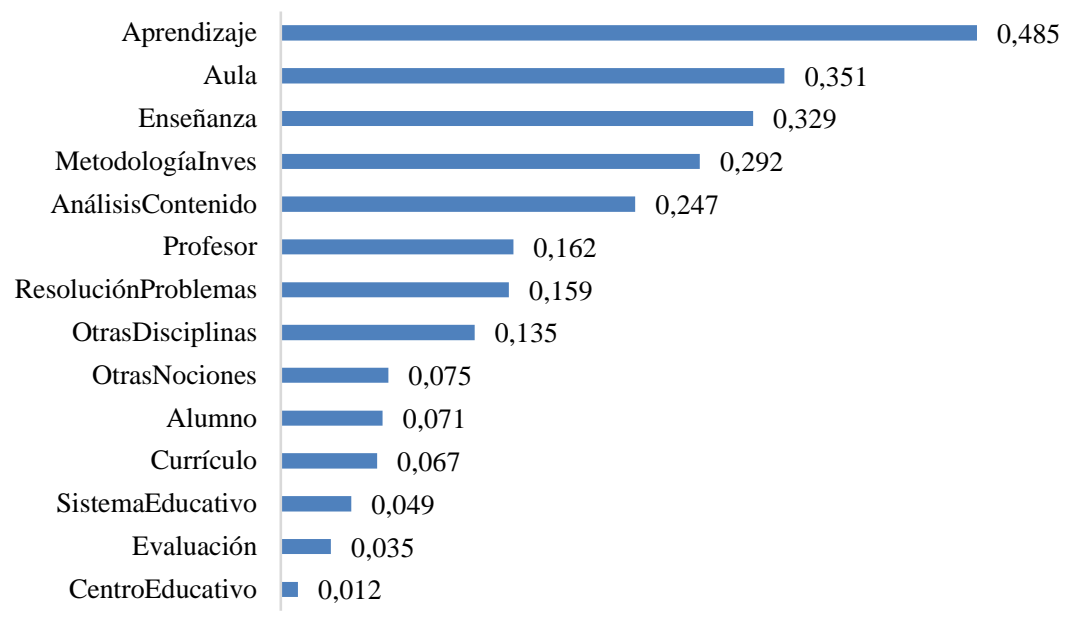

Figura 3 - Índice de especialización temática en nociones pedagógicas Fuente: elaborada por los autores

Como se aprecia en la Figura 3, la noción pedagógica que más se trata en la comunidad es aprendizaje, seguida de las nociones aula y enseñanza. La noción asociada a metodología de investigación en Educación Matemática está por encima de nociones como alumno, currículo y evaluación. Por ejemplo, el índice de esta última noción es cercano a la octava parte del índice que tiene metodología de investigación y a la mitad del índice de la noción alumno. El análisis de contenido matemático (historia, sistemas de representación y fenomenología) tiene una importancia destacable en la comunidad. Esta noción tiene el quinto mayor índice.

En la muestra, se destaca la cantidad de documentos de la disciplina que trata cuestiones asociadas a cualquier tema de matemáticas. Es en la variable cualquier tema en la que esta comunidad tiene el mayor índice de especialización (Figura 4). El tema de matemáticas con mayor índice es geometría. Le siguen los temas álgebra y números. La medida es el tema menos tratado en la documentación analizada en este estudio.

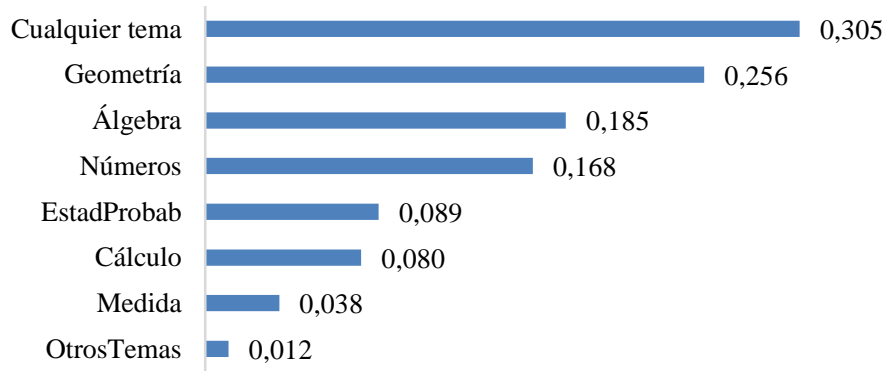

Figura 4 - Índice de especialización temática en temas de matemáticas Fuente: elaborada por los autores 


\subsection{Comportamiento diacrónico de los contenidos}

En relación con el comportamiento diacrónico de los contenidos, no identificamos grandes variaciones en la medida en la que se tratan las variables de los conjuntos nivel educativo, nociones pedagógicas y temas de matemáticas. En los diferentes conjuntos de variables, vemos que las especializaciones temáticas que presentamos en la sección anterior se conservan en el tiempo. A partir de 1994, año en el que finaliza la etapa de precursores, se empieza a estabilizar la distribución porcentual de las variables por año. En general, las series temporales correspondientes a cada variable no evidencian una tendencia. Las proporciones de documentos por año oscilan alrededor de la media de documentos producidos entre 1983 y 2017, pero este comportamiento es más evidente desde 1994. Como ejemplo, presentamos en la Figura 5 la serie temporal de la variable aprendizaje.

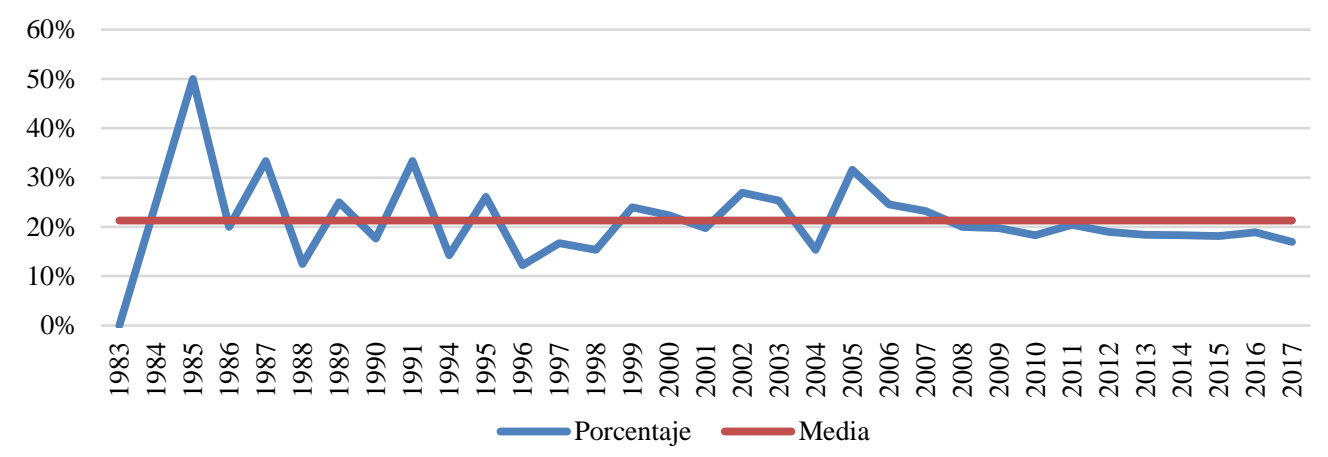

Figura 5 - Serie temporal de la variable aprendizaje Fuente: elaborada por los autores

Aplicamos la prueba Phillips-Perron (PP) para confirmar que las series temporales correspondientes a cada variable del estudio son estacionarias entre los años 1994 y 2017. Los P-valor obtenidos en las pruebas de hipótesis nos permiten afirmar que existe suficiente evidencia muestral que indica que las series temporales diferentes a centro educativo, alumno, otras nociones pedagógicas y cualquier tema de matemáticas, son estacionarias en media. Para dichas series temporales, los estadísticos de prueba son menores que los valores críticos MacKinnon, por lo que los $\mathrm{P}$-valor fueron mayores a 0,01 ( $\mathrm{P}=$ 0,2193, 0,6407, 0,0363 y 0,0138, respectivamente).

Para ampliar el análisis del comportamiento diacrónico de los contenidos, en términos de las variables del estudio, describimos algunas características de los conjuntos de variables en el tiempo. En la Figura 6, representamos el comportamiento diacrónico de las variables del conjunto nivel educativo. Los niveles educativos que más se trataron en la documentación entre 1994 y 2017 son pregrado, educación media, secundaria y primaria. Aunque los trabajos 
relacionados con preescolar son bajos, este nivel educativo se aborda de manera constante en el tiempo. A partir de 2008, se percibe un incremento en la medida en la que se tratan cuestiones de formación continua/técnica y posgrado.

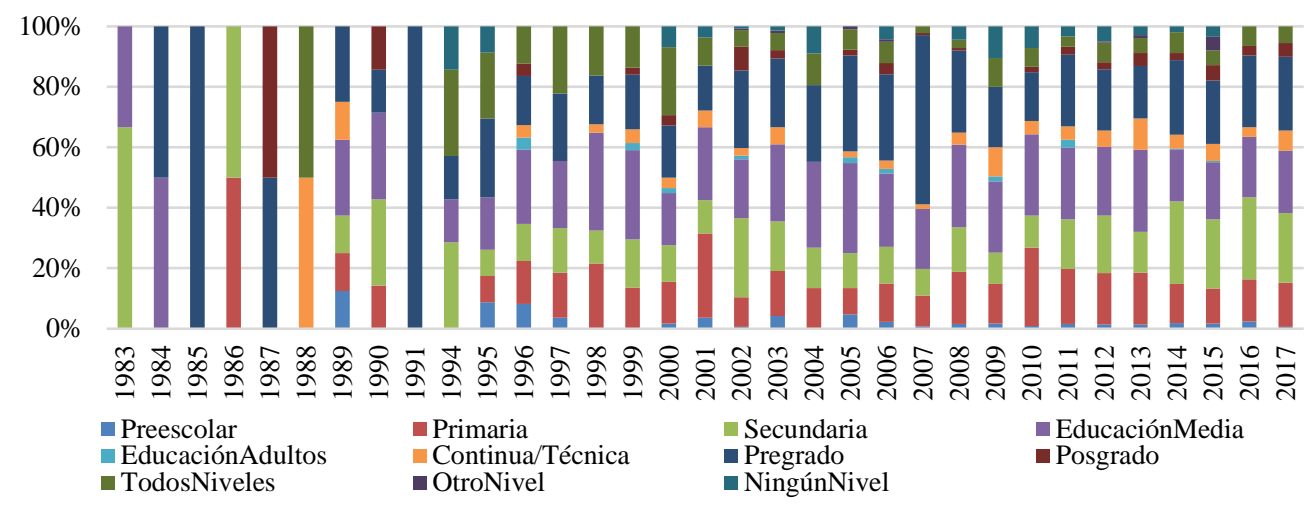

Figura 6 - Comportamiento diacrónico de los niveles educativos Fuente: elaborada por los autores

El comportamiento diacrónico de las nociones pedagógicas ratifica el interés que la comunidad colombiana de Educación Matemática tiene por cuestiones de aprendizaje, aula, enseñanza, metodología de investigación y análisis de contenido. Entre 1994 y 2017, no se perciben grandes variaciones en el porcentaje de documentos de la muestra que trata cada una de estas nociones. Si bien se produce un porcentaje pequeño de trabajos relacionados con sistema educativo, evaluación, currículo y resolución de problemas, estas nociones se han tratado, de manera continua, desde el año 2000. Cuestiones asociadas a la noción alumno, aunque en poca medida, empiezan a tratarse, de manera ininterrumpida, a partir del año 2006.

Encontramos que, a partir del año 2000, se evidencia continuidad en la proporción de documentos que tratan temas específicos de matemáticas (álgebra, cálculo, estadística y probabilidad, geometría, medida y números). Entre los años 1994 y 2000, el mayor porcentaje de documentos trata cuestiones asociadas a cualquier tema de matemáticas. Entre 2001 y 2010, la proporción promedio de documentos en la variable denominada cualquier tema es del $24 \%$. No obstante, a partir del año 2011, hay un aumento en la documentación asociada a esta variable. La distribución porcentual de la documentación por año confirma el interés que la comunidad colombiana de Educación Matemática tiene por los temas geometría, álgebra y números.

\subsection{Asociación entre conjuntos de variables}

Realizamos pruebas de independencia entre los conjuntos de variables del estudio. De acuerdo con el P-valor obtenido, afirmamos que existe suficiente evidencia muestral para 
confirmar que los conjuntos de variables nivel educativo, nociones pedagógicas y temas de matemáticas no son independientes en la documentación de acceso abierto de la comunidad colombiana de Educación Matemática. Presentamos, en el Cuadro 3, los P-valor que resultaron de las pruebas de independencia para cada asociación de conjuntos de variables.

\begin{tabular}{|l|c|}
\hline \multicolumn{1}{|c|}{ Asociaciones de conjuntos de variables } & P-valor \\
\hline Nivel educativo y nociones pedagógicas & $1,89 \mathrm{E}-115$ \\
Nivel educativo y temas de matemáticas & $2,15 \mathrm{E}-177$ \\
Nociones pedagógicas y temas de matemáticas & $7,02 \mathrm{E}-126$ \\
\hline
\end{tabular}

Cuadro 3 - P-valor en pruebas de independencia de los conjuntos de variables Fuente: elaborado por los autores

\subsection{Resumen de los resultados}

En línea con los objetivos del estudio, logramos caracterizar la comunidad colombiana de Educación Matemática en términos del comportamiento diacrónico de su documentación, sus focos de interés, la evolución de estos focos en el tiempo y la asociación de los niveles educativos, las nociones pedagógicas y los temas de matemáticas.

Los resultados del estudio confirman el crecimiento exponencial de la documentación de Educación Matemática en Colombia, lo que indica que esta disciplina se consolida como un frente de estudio. Los focos de interés de la comunidad están en los niveles educativos pregrado, media, secundaria y primaria. Además, se tratan en mayor medida las nociones aprendizaje, aula, enseñanza y metodología de la investigación en Educación Matemática. La mayoría de los documentos no trata temas específicos de matemáticas; no obstante, los temas geometría, álgebra y números se tratan de manera importante en la documentación analizada.

A partir de 1994, año en el que la producción documental pasa de la etapa de precursores a la de crecimiento exponencial, se estabiliza la distribución porcentual de las variables en el tiempo. En los niveles educativos, las nociones pedagógicas y los temas de matemáticas se confirman los intereses de la comunidad por la educación media, el aprendizaje y la geometría, como ejemplo. La medida en la que los focos de interés son tratados por la comunidad no cambia en el tiempo: las variables se comportan como series temporales estacionarias, con excepción de centro educativo, alumno, otras nociones pedagógicas y cualquier tema de matemáticas. Por último, existe asociación entre los conjuntos de variables nivel educativo, nociones pedagógicas y temas de matemáticas. 


\section{Conclusiones}

Identificamos las características de la comunidad de investigadores e innovadores en Educación Matemática de Colombia a partir de su documentación de acceso abierto. Realizamos una aproximación semántica al contenido de los documentos producidos entre 1983 y 2017. Aunque los estudios cienciométricos usualmente se centran en los artículos de investigación, tuvimos en cuenta diversas fuentes de información que merecen ser investigadas (CALLON et al., 1995). Con base en una taxonomía específica de la disciplina, establecimos los conjuntos de variables del estudio con los que codificamos los documentos.

Determinamos el comportamiento diacrónico de la producción documental de la comunidad con el propósito de verificar la ley de crecimiento exponencial. También, determinamos los índices de especialización temática de la documentación en las variables de los conjuntos nivel educativo, nociones pedagógicas y temas de matemáticas. Con ello, establecimos los focos de interés de la comunidad. Identificamos el comportamiento diacrónico de los contenidos en términos de las variables del estudio. Por último, empleamos tablas de contingencia para relacionar los conjuntos de variables y determinar si son o no independientes. De esta manera, cumplimos los objetivos específicos del estudio y proporcionamos los atributos de caracterización de la comunidad que proporcionamos en el resumen de los resultados.

Este estudio resulta novedoso en diferentes sentidos. Hasta ahora, no se identifican estudios cienciométricos sistemáticos de la Educación Matemática en Colombia y no se ha verificado que esta disciplina es un frente de estudio en el país. En relación con los estudios documentales realizados en Educación Matemática, nuestro trabajo, aunque vincula la verificación de la ley de crecimiento de la documentación, busca profundizar en el contenido de los documentos. Realizamos una aproximación semántica para determinar cuáles son los temas tratados por la comunidad, cómo es la evolución de los contenidos y establecer si hay dependencia entre, por ejemplo, los niveles educativos y las nociones pedagógicas que se abordan. La identificación y asignación de términos clave, asociados a las variables propuestas, no surge del conteo de palabras, ni se limita a la descripción de la información que se proporciona en el título o resumen de los documentos.

Por otra parte, las fuentes de información de los estudios cienciométricos en Educación Matemática han sido revistas y eventos de investigación, bases de datos como Scopus o Web of Science y tesis doctorales. Esto implica que documentación que surge de ensayos e innovaciones curriculares se ha dejado de lado en el esfuerzo por caracterizar la comunidad. En este estudio, atendemos a la diversidad de la documentación que produce la comunidad, por lo 
que trabajamos con documentos de acceso abierto entre los que se incluyen artículos publicados en revistas de divulgación, contribuciones a memorias de eventos, trabajos de grado de pregrado, libros, capítulos de libro, documentos de trabajo, entre otros. No establecemos en este documento las posibles relaciones de dependencia que puedan surgir entre los tipos de trabajos que son producidos en la disciplina. No obstante, sí consideramos que al análisis de, por ejemplo, el comportamiento diacrónico de la cantidad de ensayos y la cantidad de innovaciones como una oportunidad de investigación. Este aspecto podría dar cuenta de la evolución de la formalización de las posturas generadas por la comunidad.

El trabajo que realizamos proporciona un esquema metodológico que permite caracterizar diversas comunidades de disciplinas académicas. Genera nuevas oportunidades de investigación para la comunidad de Educación Matemática. En primer lugar, se podrían definir algunas subvariables para especificar los intereses de la comunidad en lo que respecta, por ejemplo, a aprendizaje, que es la noción pedagógica con mayor índice de especialización temática. En futuros estudios, sería interesante indagar por asociaciones temáticas en la documentación; esto es, establecer en qué medida se tratan las nociones pedagógicas y los temas de matemáticas en los niveles educativos, o las nociones pedagógicas en los temas de matemáticas. Consideramos que también hay posibilidad de comparar instituciones (programas académicos o grupos de investigación) en relación con los documentos que producen. Existe la posibilidad de caracterizar los aportes que se hace a la disciplina desde los programas de pregrado de formación de profesores y en las regiones.

Este estudio pone en evidencia la necesidad de caracterizar la Educación Matemática a partir de la diversidad de su documentación, no solo desde artículos de investigación. Dada la naturaleza de esta disciplina, así como es relevante la difusión de los avances de los investigadores, es importante que los profesores sean innovadores, compartan y debatan sus trabajos con colegas y expertos. Por ello, queda abierta la discusión en la comunidad de investigadores y educadores matemáticos respecto a cómo considerar que la Educación Matemática está consolidada como disciplina en términos de la relación entre la proporción de documentos de investigación y de innovación.

\section{Agradecimientos}

Este trabajo se realizó con el apoyo de la Facultad de Educación y la Vicerrectoría de Investigaciones de la Universidad de los Andes (Colombia) -PDI-CIFE 2016-2020 - y del 
Departamento Administrativo de Ciencia, Tecnología e Innovación - Colciencias - proyecto código 80740-179-2019.

\section{Referencias}

ARDANUY, J. Breve introducción a la bibliometría. Barcelona: Universitat de Barcelona, 2012.

ARENCIBIA, R.; DE MOYA, F. La evaluación de la investigación científica: una aproximación teórica desde la cienciometría. ACIMED, La Habana, v. 17, n. 4, p. 1-27, 2008.

BONILLA, M.; OBANDO, G. ASOCOLME - 20 años. En: ENCUENTRO COLOMBIANO DE MATEMÁTICA EDUCATIVA (ECME) 17, 2018, Bogotá.

BRACHO, R., et al. Tendencias Temáticas de la Investigación en Educación Matemática en España. Bolema, Rio Claro, v. 28, n. 50, p. 1077-1094, dic. 2014.

CALLON, M., et al. Cienciometría: El estudio cuantitativo de la actividad científica: de la bibliometría a la vigilancia tecnológica. Gijón: Ediciones TREA, 1995.

CASTRO, P.; GÓMEZ, P. Análisis bibliométrico de las memorias del Encuentro de Geometría y sus Aplicaciones entre 2002 y 2015. En: ENCUENTRO DE GEOMETRÍA Y SUS APLICACIONES, 23., 2017, Bogotá. Actas... Bogotá: Universidad Pedagógica Nacional, 2017. p. 3-10.

ERNEST, P. A postmodern perspective on research in mathematics education. En: SIERPINSKA, A.; KILPATRICK, J. (ed.). Mathematics education as a research domain: A search for identity. Dordrecht: Springer, 1998. p. 71-85.

ESCORCIA, T. El análisis bibliométrico como herramienta para el seguimiento de publicaciones científicas, tesis y trabajos de grado. 2008. Documento de Conclusión de la Carrera (Graduación en Microbiología Industrial) - Facultad de Ciencias, Pontificia Universidad Javeriana, Bogotá, 2008.

FERNÁNDEZ-CANO, A.; BUENO-SÁNCHEZ, A. Síntesis de estudios bibliométricos españoles en educación. Una dimensión evaluativa. Revista española de documentación científica, Madrid, v. 21, n. 3, p. 269-285, 1998.

FIZ KARLSRUHE. MathEduc Database. 2010. Disponible en: http://www.zentralblattmath.org/matheduc/classification/. Acceso en: 10 feb. 2017.

GÓMEZ, P.; CAÑADAS, M. C. Development of a taxonomy for key terms in mathematics education and its use in a digital repository. Library Philosophy and Practice (e-journal), 2013.

GÓMEZ, P., et al. Análisis temático de la investigación en Educación Matemática en España a través de los simposios de la SEIEM. En: MARÍN, M., et al (ed.). Investigación en Educación Matemática XV. Ciudad Real: SEIEM, 2011. p. 371-382.

KRIPPENDORFF, K. Metodología de análisis de contenido: Teoría y práctica. España: 
Paidós, 1990.

LÓPEZ-PIÑERO, J. M.; TERRADA-FERRANDIS, M. L. Los indicadores bibliométricos y la evaluación de la actividad médico-científica: los indicadores de producción, circulación y dispersión, consumo de la información y repercusión. Medicina clínica, Barcelona, v. 98, n. 4, p. 142-148, 1992.

MACÍAS-CHAPULA, C. A. Papel de la informetría y de la cienciometría y su perspectiva nacional e internacional. ACIMED, La Habana, v. 9, n. 4, p. 35-41, 2001.

MAZ, A., et al. Los Simposios de la Sociedad Española de Investigación en Educación Matemática: Una revisión bibliométrica. En: GONZÁLEZ, M. J., et al (ed.). Investigación en Educación Matemática XIII. Santander: SEIEM, 2009. p. 323-332.

MAZ-MACHADO, A., et al. Análisis bibliométrico de la revista RELIME (1997-2011). Investigación Bibliotecológica, México, v. 29, n. 66, p. 91-104, 2015.

MEDINA, J. M. La investigación odontológica en la base Science Citation Index: un estudio cienciométrico (1974-2003). 2005. Tesis/Dissertación (Doctorado en Odontología). Universidad de Granada, Granada, España, 2005.

MILLÁN, J. D., et al. La cienciometría, su método y su filosofía: Reflexiones epistémicas de sus alcances en el siglo XXI. Revista Guillermo de Ockham, Cali, v. 15, n. 2, p. 17-27, 2018.

MUÑOZ-REPISO, M. Investigación, política y prácticas educativas. REICE, Madrid, v. 8, n. 2, p. 201-216, 2010.

NATIONAL INFORMATION STANDARDS ORGANIZATION. Guidelines for the construction, format, and management of monolingual controlled vocabularies. Bethesda: NISO Press, 2005.

PÉREZ-ANGÓN, M. Á. Usos y abusos de la cienciometría. Cinvestav, México, v. 25, n. 1, p. 29-33, 2006.

PRICE, D. J. Hacia una ciencia de la ciencia. Barcelona: Editorial Ariel, 1973

ROMBERG, T. A. Perspectives on scholarship and research methods. En: GROUWS, D. (ed.). Handbook of research on the teaching and learning of mathematics. New York: Macmillan, 1992. p. 49-64.

SANCHO, R. Indicadores bibliométricos utilizados en la evaluación de la ciencia y la tecnología. Revisión bibliográfica. Revista española de documentación científica, Madrid, v. 13, n. 3-4, p. 842-865, 1990.

SOLANO, E., et al. La bibliometría: una herramienta eficaz para evaluar la actividad científica postgraduada. MediSur, Cienfuegos, v. 7, n. 4, p. 59-62, 2009.

SPINAK, E. Diccionario enciclopédico de bibliometría, cienciometría e informática. Caracas: UNESCO, 1996 
SPINAK, E. Indicadores cienciométricos. ACIMED, La Habana, v. 9, n. 4, p. 16-18, 2001.

VALLEJO-RUIZ, M., et al. Patrones de citación en la investigación española en educación matemática. Revista española de documentación científica, Madrid, v. 29, n. 3, p. 382-397, 2006.

WALDEGG, G. La educación matemática ¿una disciplina científica? Colección Pedagógica Universitaria, Veracruz, v. 29, p. 13-44, 1998.

Submetido em 04 de Dezembro de 2019. Aprovado em 16 de Maio de 2020. 